\title{
A SOLUTION TO A PROBLEM OF EILENBERG CONCERNING DIMENSION LOWERING MAPPINGS
}

\author{
JAMES KEESLING ${ }^{1}$ AND DAVID C. WILSON
}

\begin{abstract}
This paper describes a map $f$ from a metric space $X$ (having the same dimension at each of its points) onto a space $Y$ such that $\operatorname{dim} X>$ $\operatorname{dim} Y>0$ with the property that $\operatorname{dim} K \geq \operatorname{dim} f(K)$ for every closed set $K$ contained in $X$. This answers a question posed by Eilenberg in 1936 in The Scottish Book. This question was answered with a more complicated example by Rubin, Schori, and Walsh.
\end{abstract}

In 1936 Eilenberg posed the following problem in The Scottish Book [10, Problem \#137]. If $f$ maps a compact metric space $X$ (having the same dimension at each of its points) onto $Y$ such that $\operatorname{dim} X>\operatorname{dim} Y>0$, must there be a closed subset $K$ contained in $X$ such that $\operatorname{dim} K<\operatorname{dim} f(K)$ ? He also asked about the special case that $X$ is the unit square and $Y$ is the unit interval. In [7] Kelley settled this special case by showing that there is always a closed set $K$ contained in $I^{2}$ with $\operatorname{dim} K=0$ such that $f(K)=I$, Kelley attributes this result to Eilenberg and Zippin. Recently Mahavier [8] improved on this particular result.

In [7] Kelley showed that if $f(X)=Y$ is open and monotone with nondegenerate point inverses and $Y$ is finite-dimensional, then there is a closed 0-dimensional $K$ in $X$ with $f(K)=Y$. Civin and Roberts [3] and Keesling [5, 6] generalized this theorem of Kelley. Anderson [1] pointed out that Bing's examples of higherdimensional hereditarily indecomposable continua [2] together with certain results of Kelley in [7] show that the finite-dimensionality of $Y$ is necessary in this result. These results imply that a function giving a negative answer to the problem of Eilenberg cannot be monotone and open.

This paper describes examples which give a negative answer to Eilenberg's problem. For each $n \geq 2$ we construct a continuum $X$ which has dimension $n$ at each of its points and a map $f$ of $X$ onto the unit interval $I$ such that $f$ does not raise the dimension of any closed subset. The space $X$ is a closed subset of the $(n+1)$-cube and $f$ is the restriction to $X$ of the projection onto one coordinate. For each $n, f$ is monotone and in fact cell-like. The point inverses of $f$ are either $n$-cells or arc-like (chainable) continua. Of course $f$ is not an open mapping by the above remarks.

A complicated example answering Eilenberg's question can be found in Rubin, Schori, and Walsh [9]. In that paper a continuum $X$ is constructed which has the property that any closed finite-dimensional $K$ in $X$ is 0 -dimensional. In the construction of $X$ it is demonstrated that there is a map $f: X \rightarrow I$ such that any

Received by the editors September 28, 1981.

1980 Mathematics Subject Classification. Primary 54F45; Secondary 54C99.

Key words and phrases. Dimension, mapping, monotone mapping, cell-like mapping, dimension lowering mapping.

${ }^{1}$ This material was presented by the first author at the Spring Topology Conference held at the U.S. Naval Academy in Annapolis, Maryland, in March 1982. 
closed 0-dimensional set $K$ in $X$ has 0-dimensional image in $I$. This gives a negative answer to Eilenberg's general question although the authors did not seem to be aware of that fact. The examples given here are simpler and more straightforward. Also, the maps we construct are cell-like which was not the case in [9].

If $A$ is contained in $X$, then the boundary of $A$ will be denoted by $\operatorname{Fr}(A)$.

MAIN THEOREM. If $n \geq 2$, then there is an $n$-dimensional continuum $X$ contained in $I^{n+1}$ and a map $f: X \rightarrow I$ such that if $K$ is any 0-dimensional compactum in $X$, then $f(K)$ is 0-dimensional. Moreoever, the continuum $X$ is $n$-dimensional at each of its points and the map $f$ is the restriction to $X$ of the projection map $p: I^{n+1} \rightarrow I$ onto the last coordinate.

Proof. Let $\left\{r_{1}, r_{2}, \ldots\right\}$ be a countable dense subset of $I$. The space $X$ will be obtained as the nested intersection of continua $X_{0}, X_{1}, \ldots, X_{k}, \ldots$ Let $X_{0}=$ $I^{n+1}, U_{0}=\emptyset$, and $p_{k}$ denote the restriction of $p$ to the set $X_{k}$.

We define the following two subsets of $I^{n}$ : the "back" face, $B=\left\{\left(0, x_{2}, \ldots, x_{n}\right) \mid\right.$ $\left.x_{i} \in I\right\}$, and the "front" face, $F=\left\{\left(1, x_{2}, \ldots, x_{n}\right) \mid x_{i} \in I\right\}$.

Assume inductively that we have found continua $X_{0}, X_{1}, \ldots, X_{k}$ and open sets $U_{0}, U_{1}, \ldots, U_{k}$ with the following properties. (1) If $j>0$, then $U_{j}$ is an open subset of $I$ with diameter less than $1 / j$ which is the union of a countable sequence of pairwise disjoint open intervals which converge to $r_{j}$. (2) If $i \neq j$, then $r_{i} \notin \operatorname{Fr}\left(U_{j}\right)$ and $\operatorname{Fr}\left(U_{i}\right) \cap \operatorname{Fr}\left(U_{j}\right)=\emptyset$. (3) If $i<j$, then either $\bar{U}_{i} \cap \bar{U}_{j}=\emptyset$ or $\bar{U}_{j}$ is contained in one component of $U_{i}$. (4) $X_{k} \subset X_{k-1} \subset \cdots \subset X_{1} \subset X_{0}=I^{n+1}$. (5) The set $p_{j}^{-1}(y)$ is an $n$-cell for all $Y \in I$. (6) The set $p_{j-1}^{-1}\left(I-U_{j}\right)=p_{j}^{-1}\left(I-U_{j}\right)$. (7) If $U_{j}(i)$ is a component of $U_{j}$, then there is a polyhedral $n$-cell $N_{j}(i) \subseteq I^{n}$ with the property that $p_{j}^{-1}\left(U_{j}(i)\right)=N_{j}(i) \times U_{j}(i)$. (8) Each $n$-cell $N_{j}(i)$ meets both the set $B$ and the set $F$ in an $(n-1)$-cell. (9) If $A$ is any arc from $B$ to $F$ in $p_{j}^{-1}\left(r_{j}\right)$, then for each $\epsilon>0$ there is an integer $i$ such that $N_{j}(i)$ is contained in the $\epsilon$-neighborhood of $A$.

Note that $U_{0}$ and $X_{0}$ satisfy properties (1) through (9). While there is a lot of properties to check in the induction statement, the authors would like to point out that properties (1) to (5) and (8) are included to guarantee that the set $X_{j}$ will "look like" the set $X_{j-1}$. Property (6) assures that when constructing $X_{j}$ from $X_{j-1}$, the only changes to be made will be over the set $U_{j}$. Property (7) assures that $X_{j}$ will look like a product over the components of $U_{j}$. Property (9) states that as the components $U_{j}(i)$ of $U_{j}$ converge to $r_{j}$, the set of $n$-cells $N_{j}(i)$ are dense in the set of arcs in $p_{j}^{-1}\left(r_{j}\right)$ which go from "back" to "front".

We now want to show the existence of sets $X_{k+1}$ and $U_{k+1}$ which satisfy properties (1) to (9) above. First find points $a$ and $b$ such that $a<r_{k+1}<b$ and the interval $[a, b]$ misses the set $\left\{r_{1}, r_{2}, \ldots, r_{k}\right\}$. By properties (2) and (3) we can also assume that if $[a, b]$ meets a set $U_{j}$, then it is contained in some component of $U_{j}$. Inside $(a, b)$ now find a small open set $U_{k+1}$ which is the union of a countable sequence of pairwise disjoint open intervals which converge to $r_{k+1}$. By properties (6) and (7) we know that $p_{k}^{-1}((a, b))=N \times(a, b)$ for some $n$-cell $N$. Let $A_{1}, A_{2}, \ldots$ be a sequence of polyhedral arcs in $N$ which begin in $B$ and end in $F$ and which are dense (in the hyperspace topology) in the collection of all arcs in $N$ which begin in $B$ and end in $F$. Let $N_{k+1}(i)$ be a regular neighborhood of $A_{j}$ which is contained in the $1 / i$-neighborhood of $A_{i}$ and which meets $B$ and 
$F$ in $(n-1)$-cells. If $U_{k+1}=\bigcap_{i=1}^{\infty} U_{k+1}(i)$, where the set $U_{k+1}(i)$ is the $i$ th component of $U_{k+1}$ converging to $r_{k+1}$, then define the set $X_{k+1}$ above $U_{k+1}(i)$ (i.e., $\left.p_{k+1}^{-1}\left(U_{k+1}(i)\right)\right)$ to be the set $N_{k+1}(i) \times U_{k+1}(i)$. Above the set $I-U_{k+1}$ leave the set as it was before. More precisely, define $X_{k+1}$ above $I-U_{k+1}$ by the formula $p_{k+1}^{-1}\left(I-U_{k+1}\right)=p_{k}^{-1}\left(I-U_{k+1}\right)$. We have now shown that the sets $X_{k+1}$ and $U_{k+1}$ satisfy properties (1) and (9) and hence have completed the induction.

Let $X=\bigcup_{k=1}^{\infty} X_{k}$ and let $f=p$ restricted to the set $X$. Let $K$ be a closed subset of $X$ with the property that $f(K)$ contains a nondegenerate interval $[a, b]$. Let $r_{k}$ be a point of the countable dense subset in $(a, b)$. We claim that $K \cap f^{-1}\left(r_{k}\right)$ contains a nondegenerate continuum and thus $K$ is not 0-dimensional. For if not, then $K \cap f^{-1}\left(r_{k}\right)=K \cap p_{k}^{-1}\left(r_{k}\right)$ is 0-dimensional. Since $n \geq 2, p_{k}^{-1}\left(r_{k}\right)$ is an $n$-cell, and $K \cap p_{k}^{-1}\left(r_{k}\right)$ is 0-dimensional, $K \cap p_{k}^{-1}\left(r_{k}\right)$ does not separate $p_{k}^{-1}\left(r_{k}\right)$. Hence, there is an arc $A$ from $B$ to $F$ in $p_{k}^{-1}\left(r_{k}\right)$ which misses $K$. Let $\epsilon>0$ be chosen to be the distance from $A$ to $K$. Choose $i$ so that $U_{k}(i) \subset(a, b)$ and the neighborhood $p_{k}^{-1}\left(u_{k}(i)\right)=N_{k}(i) \times U_{k}(i)$ lies in the $\epsilon$-neighborhood of $A$. But then $f(K)$ misses $U_{k}(i)$ which contradicts the assumption that $f(K)$ contains $[a, b]$. Thus $K$ contains a nondegenerate continuum and we can conclude that the image under $f$ of every 0 -dimensional closed set is 0-dimensional.

Since $f^{-1}\left(r_{k}\right)$ is an $n$-cell and since $X$ contains no open subset of $I^{n+1}, \operatorname{dim} X=$ $n$.

Let $x$ be any point in $X$. If $f^{-1}(f(x))$ is an $n$-cell, then $X$ is obviously $n$ dimensional at $x$. On the other hand, suppose that $f^{-1}(f(x))$ is not an $n$-cell. Then by properties (5) and (6) of the induction hypothesis it must be that $f(x)$ is an element of $U_{i_{k}}$ for an infinite subsequence of the $U_{i}$ 's. By (1), the diameters of these $U_{i_{k}}$ 's go to zero as $k$ goes to infinity. Now to show that in this case also $\operatorname{dim}_{x} X=$ $n$, consider the following. Let $W$ be an open set in $X$ containing $x$ whose closure does not meet both $F$ and $B$. Let $O$ be an open set in $I^{n+1}$ such that $O \cap X=W$ and $\operatorname{Fr}(O) \cap X=\operatorname{Fr}(W)$. Now $p(O)$ is an open set in $I$ which contains $f(x)$. Since $\operatorname{diam}\left(U_{i_{k}}\right) \rightarrow 0$ and $f(x) \in U_{i_{k}}$ for all $k$, there must be a $k$ with $\bar{U}_{i_{k}} \subset f(O)$. Thus $r_{i_{k}} \in f(O)$. Now by (3) of the induction hypothesis $p_{i_{k}}^{-1}\left(r_{i_{k}}\right)=f^{-1}\left(r_{i_{k}}\right)$ which is an $n$-cell. However, this implies that $f^{-1}\left(r_{i_{k}}\right) \cap O=f^{-1}\left(r_{i_{k}}\right) \cap W \neq \emptyset$ with $f^{-1}\left(r_{i_{k}}\right) \cap W$ missing either $B$ or $F$. Thus the set $\operatorname{Fr}(W) \cap f^{-1}\left(r_{i_{k}}\right)$ is at least $(n-1)$-dimensional. Since $W$ was an arbitrary neighborhood of $x$, we have shown that $\operatorname{dim}_{x} X=n$. Thus concludes the proof.

REMARKS. One can easily verify that the map $f$ is cell-like and in fact $f^{-1}(x)$ is either an $n$-cell or an arc-like continuum. The example described by Rubin, Schori, and Walsh [9] does not give a cell-like map. One can see directly that $f$ is not open which would contradict results of Kelley [7] and others. The set of points where $f$ is arc-like is a dense $G_{\delta}$ in $i$, but can be taken to have Lebesgue measure zero. The construction can be modified so that $X$ is infinite-dimensional with $X$ contained in $Q \times I$ and with the point-inverses of $f$ either arc-like or a copy of $Q$ and with $\operatorname{dim}_{x} X=\infty$ for all $x$ in $X$. We leave this as an exercise for the readers.

It should also be pointed out that we have actually proved that if $K$ is closed subset of $X$ with $\operatorname{dim} K \leq n-2$, then $\operatorname{dim} f(K) \leq 0$. In the construction suggested above which yields an infinite-dimensional $X$ one can construct $X$ so that if $K$ is a closed subset of $X$ which is finite-dimensional, then $\operatorname{dim} f(K)$ is 0-dimensional in I. Of course, this is true of the example of Rubin, Schori, and Walsh [9]. 


\section{REFERENCES}

1. R. D. Anderson, Some remarks on totally disconnected sections of monotone open mappings, Bull. Acad. Polon. Sci. Cl. III 4 (1956), 329-330.

2. R. H. Bing, Higher-dimensional hereditarily indecomposable continua, Trans. Amer. Math. Soc. 7 (1951), 267-273.

3. P. Civin and J. H. Roberts, Sections of continuous collections, Bull. Amer. Math. Soc. 49 (1943), 142-143.

4. J. Keesling, Mappings and dimension in general metric spaces, Pacific J. Math. 25 (1968), 277288.

5. __ Open mappings and closed subsets of the domain in general metric spaces, Proc. Amer. Math. Soc. 20 (1969) 238-245.

6. __ Mappings and dimension in metric spaces, General Topology and Appl. 2 (1972), 181-192.

7. J. L. Kelley, Hyperspaces of a continuum, Trans. Amer. Math. Soc. 52 (1942), 22-36.

8. W. S. Mahavier, Dimension raising subsets of mappings onto an arc, preprint.

9. L. Rubin, R. M. Schori and J. Walsh, New dimension-theory techniques for constructing infinite-dimensional examples, General Topology and Appl. 10 (1979), 93-102.

10. S. M. Ulam, The Scottish book, Los Alamos Scientific Laboratory Monograph LA-6832, 1957.

DEPARTMENT OF MATHEMATICS, UNIVERSITY OF FLORIDA, GAINESVILLE, FLORIDA 32611 